\title{
A systematic review and meta-analysis of the correlation between hyperuricemia and thyroid nodules in adults
}

\author{
Jingyan Hu, Ying Luo, Xuebo Lin \\ Department of Endocrinology, the Affiliated People's Hospital of Ningbo University, Ningbo, China \\ Contributions: (I) Conception and design: J Hu; (II) Administrative support: Y Luo; (III) Provision of study materials or patients: X Lin; (IV) \\ Collection and assembly of data: Y Luo; (V) Data analysis and interpretation: J Hu; (VI) Manuscript writing: All authors; (VII) Final approval of \\ manuscript: All authors. \\ Correspondence to: Jingyan Hu. Department of Endocrinology, the Affiliated People's Hospital of Ningbo University, Ningbo 315040 , China. \\ Email: hujynb@163.com.
}

\begin{abstract}
Backgroundk In recent years, thyroid nodules have gradually become one of the important factors endangering people's health, and we hope to recognize the association between hyperuricemia and thyroid nodulogenesis by this meta-analysis study.

Methods: Literatures related to hyperuricemia and thyroid nodules were searched in the Chinese biomedical databases Chinese National Knowledge Infrastructure (CNKI), VIP, Wanfang, and the Chinese Biomedical Literature Database (CBM), as well as the English databases PubMed, EMBASE, and the Cochrane Library from establishment of the databases to September 2021. Duplicate literatures were identified using Endnote X9 software and excluded. All studies were screened according to the inclusion and exclusion criteria, and the quality of the included literature was assessed. Data were recorded and metaanalyzed using the Stata 15.1 software. Odds ratios (OR) and 95\% confidence intervals (CIs) were calculated using the fixed effects or random effects models. Finally, sensitivity analyses were performed to assess the reliability of the results. Funnel plots were constructed to evaluate the publication bias of the literatures.
\end{abstract}

Results: A total of 16 articles, with a total sample size of 126,907, were included in this study. Metaanalysis showed that there was no correlation between the incidence of thyroid nodules and the presence or absence of hyperuricemia in the overall population. Interestingly, hyperuricemia was found to be a protective factor for the incidence of thyroid nodules in the male population ( $\mathrm{OR}=0.91 ; 95 \% \mathrm{CI}$ : 0.86 to $0.97 ; \mathrm{P}<0.05)$. However, hyperuricemia was identified as a risk factor for the incidence of thyroid nodules in the female population ( $\mathrm{OR}=1.14$; $95 \% \mathrm{CI}$ : 1.04 to 1.25 ; $\mathrm{P}<0.05)$.

Discussion: Serum uric acid has a bidirectional regulatory effect in different genders and female patients should be wary of secondary thyroid nodules.

Keywords: Hyperuricemia; adult thyroid nodule; correlation; meta-analysis

Submitted Oct 15, 2021. Accepted for publication Dec 17, 2021.

doi: $10.21037 / g s-21-722$

View this article at: https://dx.doi.org/10.21037/gs-21-722

\section{Introduction}

Thyroid nodules are abnormal masses in the thyroid gland that can cause serious physical and mental stress on patients. While the precise causes of thyroid nodules are unclear, it is believed to be closely related to numerous factors such as gender, age, body mass index, iodine intake, total cholesterol level, hypertension, and diabetes (1-3). Thyroid degeneration, inflammation, autoimmune problems, and neoplasms may present as thyroid nodules. Timely surgical resection is crucial to avoid significant patient morbidity and mortality. Hyperuricemia occurs when there is an excess of uric acid in the blood due to purine metabolism disorders. Complications of hyperuricemia include gout, 
uremia, gouty arthritis, tophi, gouty nephropathy, uric acid nephrolithiasis, and ocular lesions. There have been conflicting reports in the literature regarding the relationship between hyperuricemia and thyroid nodules. While some reports have suggested that hyperuricemia may be a protective factor for the incidence of thyroid nodules, others believe that it may act as a risk factor $(4,5)$. Other studies have suggested that the relationship between blood uric acid levels and the incidence of thyroid nodules varies in different populations and is dependent on factors such as age and gender. Though there have been many studies on hyperuricemia and thyroid nodules, we hope to combine the latest studies to perform a meta-analysis containing a larger sample size, to examine the correlation between serum uric acid levels and the incidence of thyroid nodules.

We present the following article in accordance with the PRISMA reporting checklist (available at https://dx.doi. org/10.21037/gs-21-722).

\section{Methods}

\section{Literature search}

A literature search was performed using the Chinese medical databases China National Knowledge Infrastructure (CNKI), VIP, Wanfang, and the Chinese Biomedical Database (CBM), as well as the English databases PubMed, EMBASE, and the Cochrane Library. The search period was from database establishment to September 2021. The following terms were used alone or in combination with the corresponding subject headings in the MeSH table: [hyperuricaemia], [hyperuricemia], [hyperuricacidemia], [uric acid or urea acid], [urate], [gout], [thyroid nodular goiter], [struma nodosa], and [solitary nodular goiter].

\section{Literature selection process}

Systematic reviews, randomized controlled trials, crosssectional studies, cohort studies, and case-control studies published in English were considered for this metaanalysis. The following inclusion criteria were applied: (I) literature in which patients had a definitive diagnosis of hyperuricemia; (II) studies in which thyroid nodules were diagnosed by thyroid B ultrasound; and (III) studies which clearly mention the mean \pm standard deviation $(\bar{x} \pm s)$ values or the odds ratio (OR) and $95 \%$ confidence interval (CI) values.

Studies were excluded if it was a single case report; did not provide the $\bar{x}_{ \pm \mathrm{S}}$ or OR and $95 \%$ CI values; the subjects had no definite diagnosis of hyperuricemia; the thyroid nodule was only diagnosed by hand palpation; there was a past history of thyroid nodules or other thyroid diseases; there were confounding factors that may affect the determination of blood uric acid levels, such as failure to fast for more than 8 hours, severe renal insufficiency, or pernicious anemia; and if the language of publication was not English.

\section{Deviation risk evaluation}

The publication bias of the cross-sectional study was evaluated using the relevant criteria detailed in the American Agency for Health Care Quality and Research (AHRQ). A total of 11 items were assessed and articles with a score of $0-3$ were considered low-quality articles, a score of 4-7 indicated medium-quality articles, and papers with a score of 8 or higher were considered high-quality articles. The Newcastle-Ottawa Scale (NOS) was used to evaluate case-control studies and cohort studies. There were 8 items, with a possible full score of 9 . Articles with a score of less than 5 were considered low-quality and studies with a score of 5 or higher were considered high-quality articles.

\section{Data extraction}

After literature retrieval, the Endnote X9 software was used for unified processing. Duplicate literatures were excluded. Two evaluators independently screened the literatures according to the inclusion and exclusion criteria by reading the titles, abstracts, and full-text articles. The following data were collated: (I) the basic information of the studies including title, author, publication date, contact address, and name of publication; (II) the basic characteristics of the study including study methods, total sample size, and number of groups; and (III) the basic characteristics of the participants including gender, age, and health status. Any disputes regarding the extraction of data and quality evaluation were resolved via discussions or by seeking the opinion of a third researcher.

\section{Handling of data loss}

In cases where insufficient data was provided, the data was obtained by calculation or linkage where possible, or the original authors were contacted for the data. If the required information could not be accessed, the literature was excluded. 
Identification of studies via databases and registers

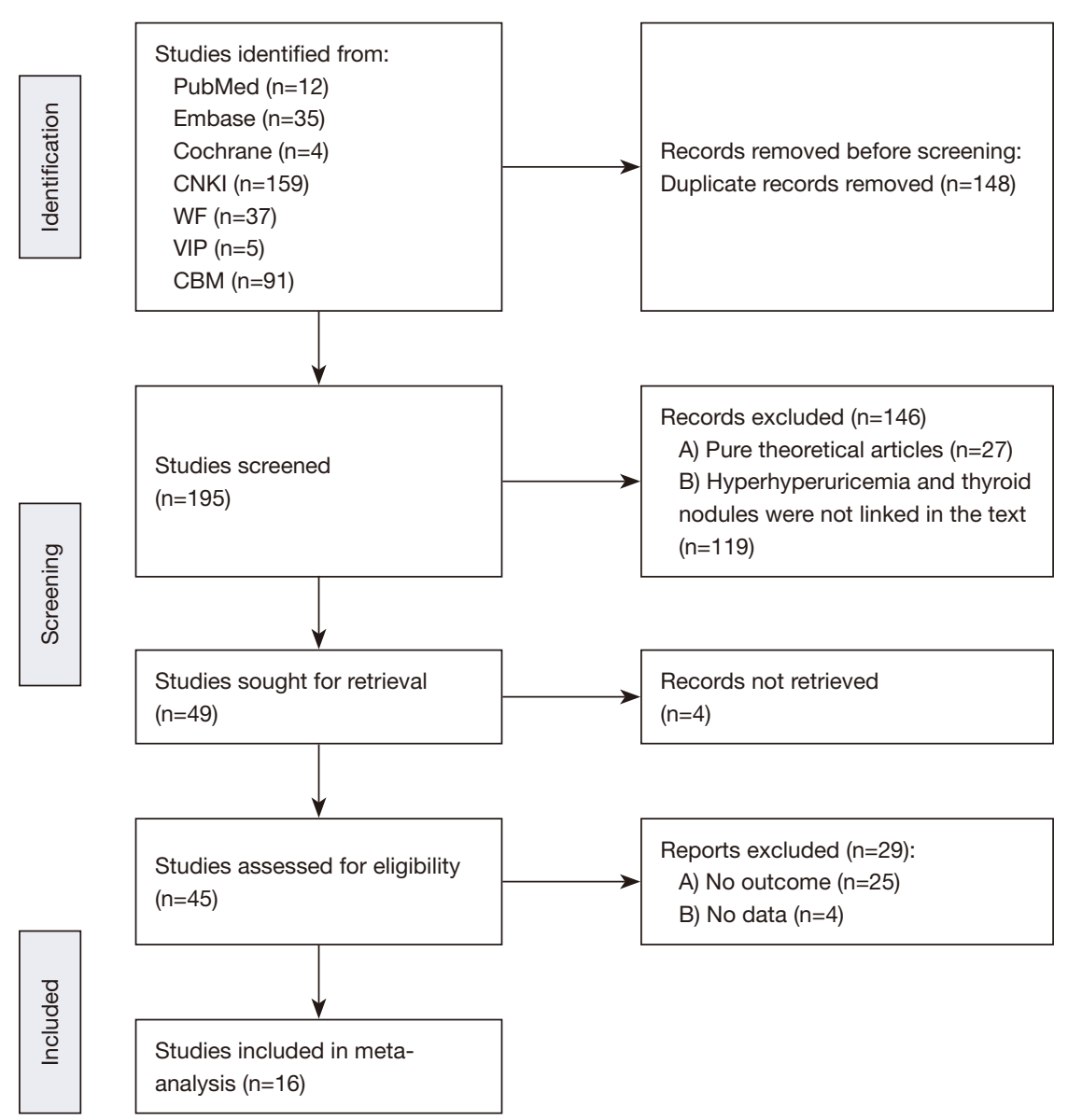

Figure 1 A flow chart showing the literature selection process.

\section{Statistical analysis}

The $\bar{x} \pm$ s, OR and the $95 \%$ CI, and forest plots were calculated using the Stata15.1 software. The $\mathrm{I}^{2}$ value was used to evaluate heterogeneity among included studies. The random effects model was used to combine study results. The AHRQ or NOS score was used for meta-analysis to determine the effects of literature quality on heterogeneity factors. Sensitivity analysis was performed by excluding any literature one by one and examining the effect of the pooled effect size. Publication bias was assessed using the funnel plot test. The OR was used for enumeration data and the standardized mean difference (SMD) was used for measurement data. Each effect size was presented with a $95 \%$ CI. When $\mathrm{A}=0.05$ or $\mathrm{P}<0.05$, the results were considered statistically significant.

\section{Results}

\section{Literature search and selection}

The database searches identified 343 articles. Exclusion of duplicate studies resulted in 195 remaining publications. A total of 146 literatures were excluded after reading the titles and abstracts. The remaining 49 publications were further screened by reading the full-text article and finally, a total of 16 articles were included in this meta-analysis. Figure 1 shows the literature search and screening process. 
Table 1 The basic characteristics of the included literatures

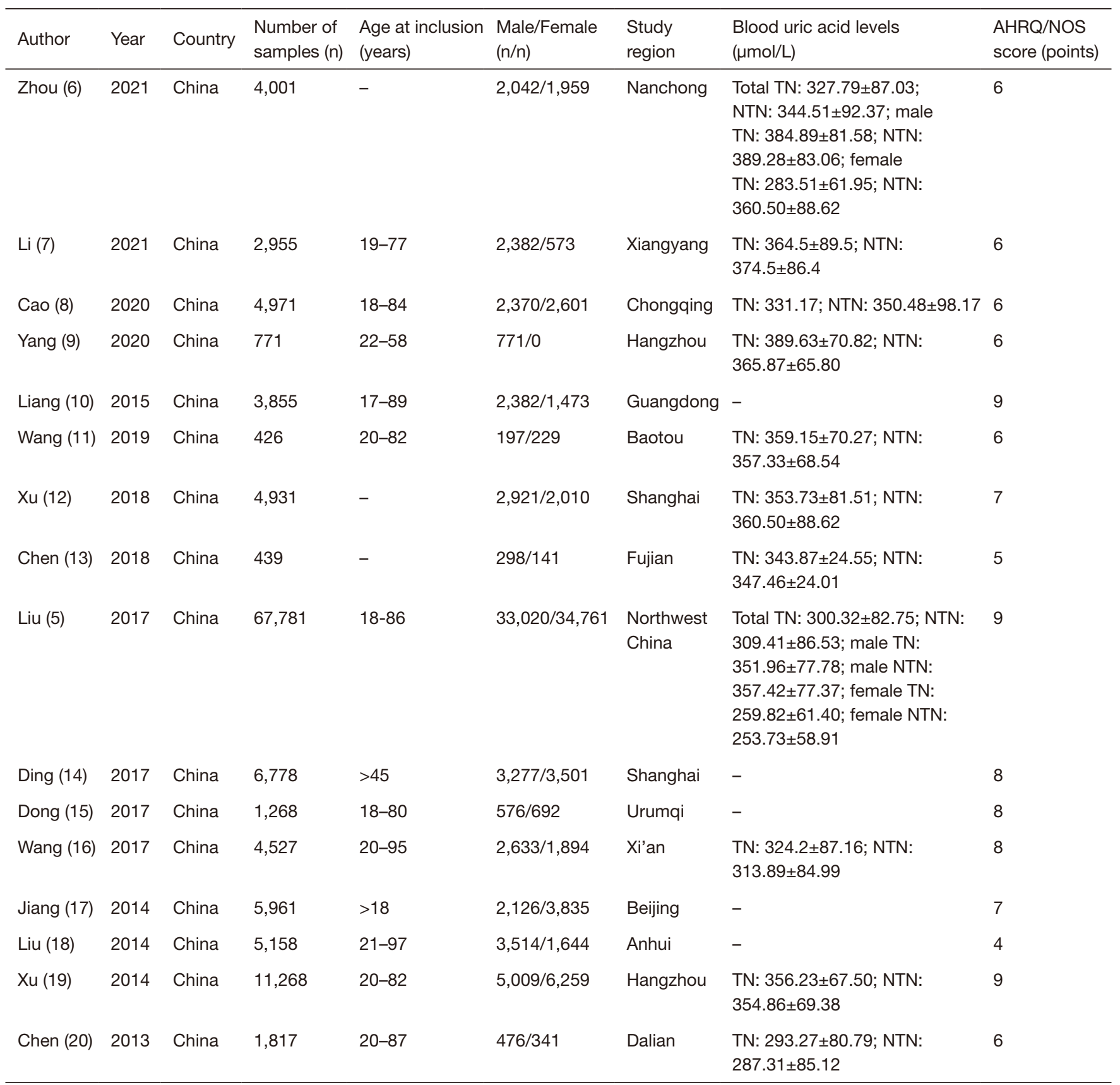

TN, thyroid nodule group; NTN, non-thyroid nodule group; -, no data.

\section{Basic characteristics of included literatures}

Meta-analysis and quality evaluation of the 16 included articles using the AHRQ and NOS scores identified 6 highquality articles and 10 medium to low quality articles. The basic information of the included studies are shown in Table 1 .

\section{The correlation between hyperuricemia and thyroid nodules in terms of odds ratios and $95 \%$ CI}

A total of 6 studies examined the relationship between hyperuricemia and thyroid nodules in terms of the OR and the $95 \%$ CI. Meta-analysis showed that hyperuricemia was not correlated with the incidence of thyroid nodules (OR 


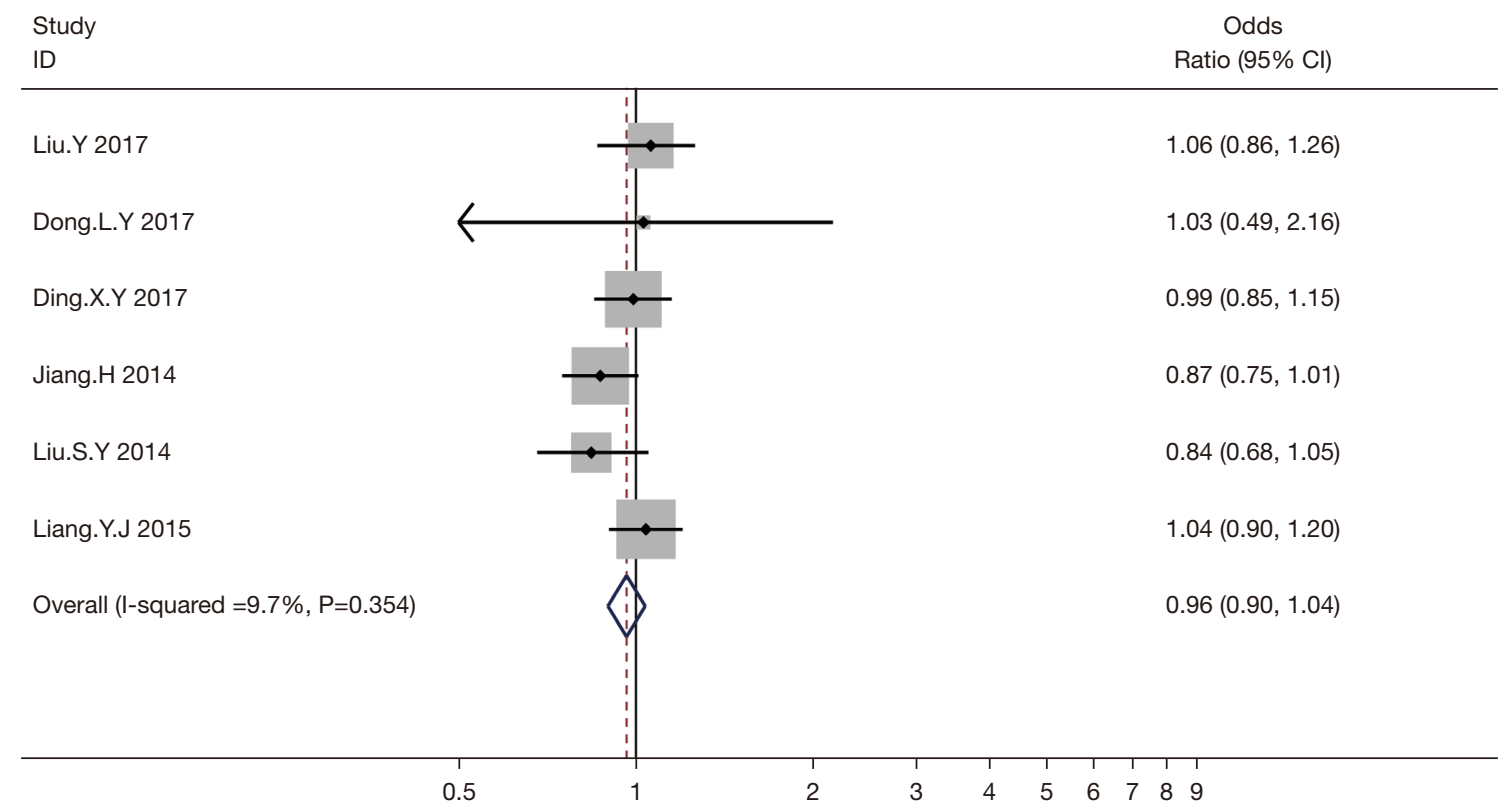

Figure 2 A forest plot showing the pooled results from studies that reported the correlation between hyperuricemia and thyroid nodules in terms of odds ratio and $95 \%$ confidence intervals.

$=0.96 ; 95 \%$ CI: 0.90 to $1.04 ;$ P>0.05; Figure 2).

\section{The correlation between hyperuricemia and thyroid nodules in terms of the mean and standard deviation}

A total of 9 articles reported the mean \pm standard deviation values of serum uric acid levels and the incidence of thyroid nodules. Meta-analysis of the combined data revealed that hyperuricemia was not associated with the incidence of thyroid nodules $(\mathrm{OR}=0.00$; $95 \% \mathrm{CI}$ : -0.09 to 0.09 ; $\mathrm{P}>0.05$; Figure 3).

\section{The correlation between hyperuricemia and thyroid nodules according to the quality of the included literature}

Meta-analysis of high-quality literatures based on AHRQ/ NOS scores demonstrated that hyperuricemia had no correlation with the incidence of thyroid nodules (OR $=0.07 ; 95 \%$ CI: -0.03 to $0.16 ; \mathrm{P}>0.05)$. Analysis of the pooled results from the medium to low quality studies also revealed that hyperuricemia had no correlation with the incidence of thyroid nodules (OR $=-0.02 ; 95 \% \mathrm{CI}:-0.13$ to 0.09; Figure 4).

\section{The correlation between hyperuricemia and thyroid nodules in males and females}

A total of 3 articles reported the effects of hyperuricemia on the incidence of thyroid nodules in males and females. Analysis of the combined results showed that hyperuricemia was a protective factor for the incidence of thyroid nodules in adult males ( $\mathrm{OR}=0.91$; 95\% CI: 0.86 to 0.97 ; $\mathrm{P}<0.05)$. However, in females, hyperuricemia was a risk factor for the incidence of thyroid nodules ( $\mathrm{OR}=1.14 ; 95 \% \mathrm{CI}: 1.04$ to 1.25 ; $\mathrm{P}<0.05$; Figure 5).

\section{Sensitivity analysis of the included literatures}

Sensitivity analysis of the 9 articles reporting mean \pm standard deviation values showed similar distribution on both sides and good stability (Figure 6).

\section{Analysis of publication bias}

Funnel plots were used to assess the publication bias in the 6 literatures that reported the OR and $95 \%$ CI of the incidence of hyperuricemia and thyroid nodules, and in the 


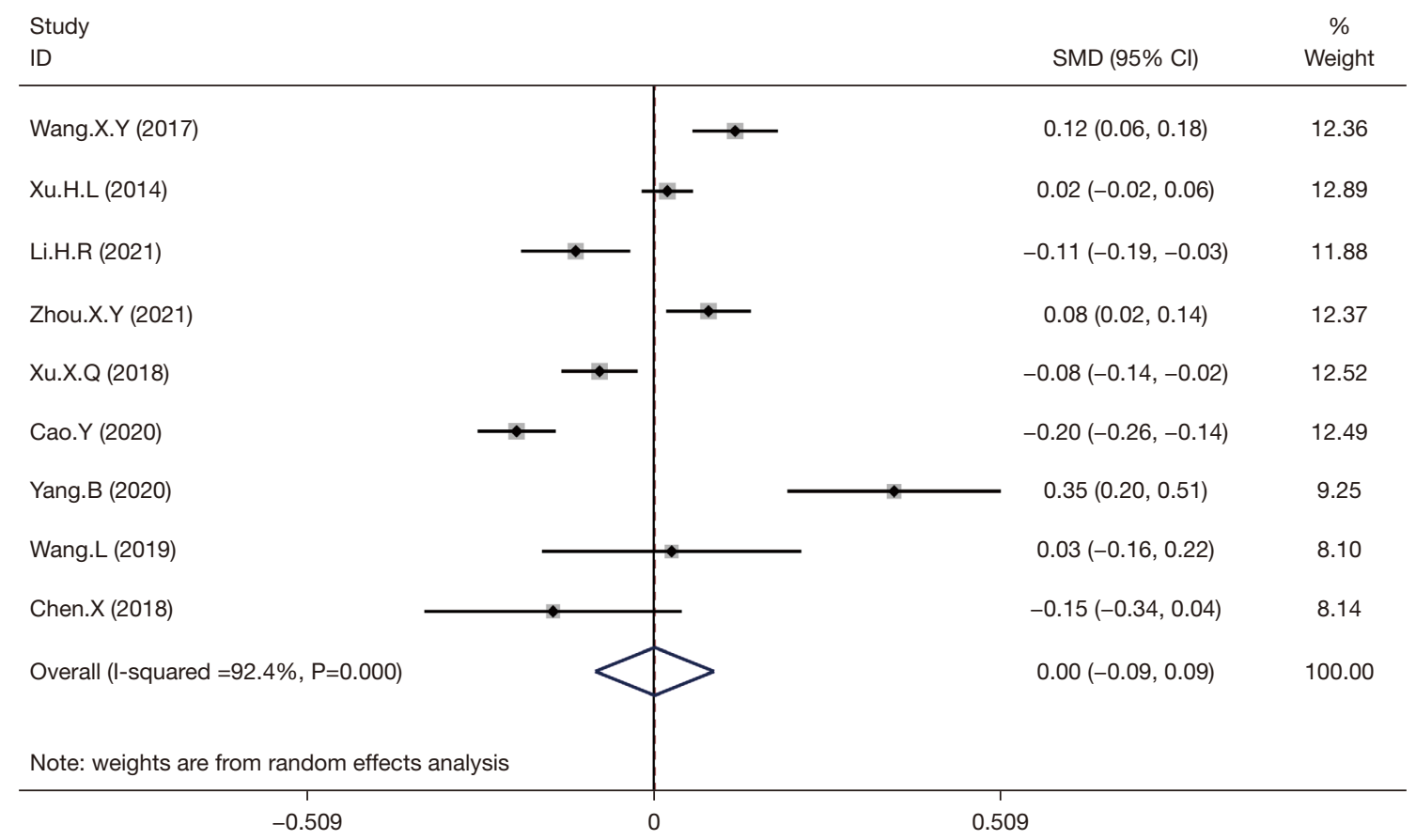

Figure 3 A forest plot showing the pooled results from studies that reported the correlation between hyperuricemia and thyroid nodules in terms of the mean and standard deviation.

9 studies that reported the mean \pm standard deviation values. The funnel plot showed that the left-right distribution of the literature was basically symmetrical without significant publication bias (Figures 7,8).

\section{Discussion}

A total of 16 articles, with a total sample size of 126,907 , were included in this meta-analysis. The overall data suggested that there was no correlation between hyperuricemia and the incidence of thyroid nodules. Interestingly, gender analysis revealed that hyperuricemia was a protective factor for the incidence of thyroid nodules in the male population $(\mathrm{OR}=0.91 ; 95 \% \mathrm{CI}$ : 0.86 to 0.97 ; $\mathrm{P}<0.05)$. Conversely, hyperuricemia was identified as a risk factor for the incidence of thyroid nodules in women (OR $=1.14 ; 95 \%$ CI: 1.04 to $1.25 ; \mathrm{P}<0.05)$. However, due to the small number of relevant studies, the low quality of the original studies, and the great variations in sample sizes, this subgroup analysis may not be satisfactory.

Hyperuricemia and thyroid nodules are relatively common diseases of the endocrine system with a high incidence rate that continues to escalate annually. However, the etiology and pathogenesis of thyroid nodules are relatively complex, and there are many relevant studies so far, but it has not been concluded. Insulin resistance syndrome is the metabolic syndrome, can cause increased serum uric acid levels. Many studies have shown that the morbidity of thyroid nodule are higher in patients with metabolic syndrome than those with non-metabolic syndrome. Liu et al. showed that longitudinal growth of serum uric acid levels in women is a risk factor for the metabolic syndrome, and this association was not found in men (21). The region with the highest prevalence of thyroid nodules in China is in Hebei Province, and the lowest is in Xinjiang Province, but the prevalence of hyperuricemia does not vary greatly between them $(22,23)$. Uric acid is a class of purine metabolites that has been shown to exert strong antioxidant effects capable of regulating metabolism and inflammation (24-28). Hyperuricemia is a significant risk factor for gout and is closely related to hypertension, type 2 diabetes, chronic kidney disease, cardiovascular disease, and metabolic syndromes (29). Interestingly, gender differences have been noted in these correlations. For example, women with hyperuricemia are more likely to experience secondary disease $(30,31)$. Consistent with this latter study, the current meta-analysis revealed that hyperuricemia is a risk factor for thyroid nodules in the female population. The incidence of 


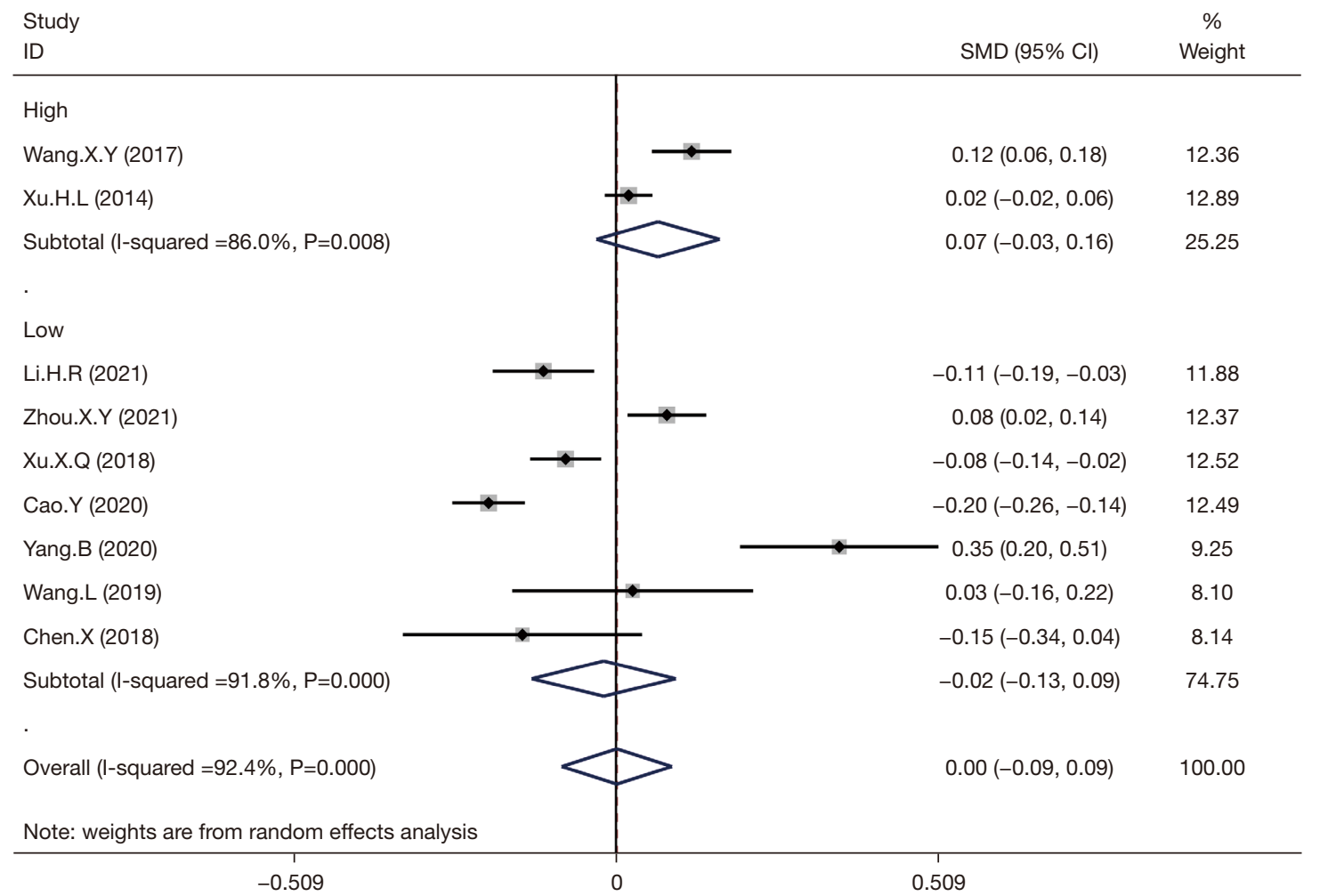

Figure 4 An analysis of the correlation between hyperuricemia and thyroid nodules in high quality studies and low to medium quality studies.

\begin{tabular}{|c|c|c|}
\hline $\begin{array}{l}\text { Study } \\
\text { ID }\end{array}$ & $\begin{array}{c}\text { Odds } \\
\text { Ratio }(95 \% \mathrm{Cl})\end{array}$ & $\begin{array}{c}\% \\
\text { Weight }\end{array}$ \\
\hline \multicolumn{3}{|l|}{ male } \\
\hline Liu.Y (2017) & $0.93(0.87,0.98)$ & 62.33 \\
\hline Ding.X.Y (2017) & $0.82(0.65,0.99)$ & 4.99 \\
\hline Jiang.H (2014) & $0.82(0.65,0.99)$ & 4.99 \\
\hline Subtotal $(I-$ squared $=15.7 \%, P=0.306)$ & $0.91(0.86,0.97)$ & 72.31 \\
\hline \multicolumn{3}{|l|}{ female } \\
\hline Liu.Y (2017) & $1.12(1.00,1.25)$ & 17.74 \\
\hline Ding.X.Y (2017) & $1.24(0.99,1.53)$ & 4.66 \\
\hline Jiang.H (2014) & $1.12(0.91,1.37)$ & 5.28 \\
\hline Subtotal (I-squared $=0.0 \%, P=0.705)$ & $1.14(1.04,1.25)$ & 27.69 \\
\hline \multicolumn{3}{|l|}{ Heterogeneity between groups: $P=0.000$} \\
\hline Overall (I-squared $=75.0 \%, P=0.001$ ) & $0.97(0.93,1.02)$ & 100.00 \\
\hline
\end{tabular}

Figure 5 An analysis of the correlation between hyperuricemia and thyroid nodules in males and females. 


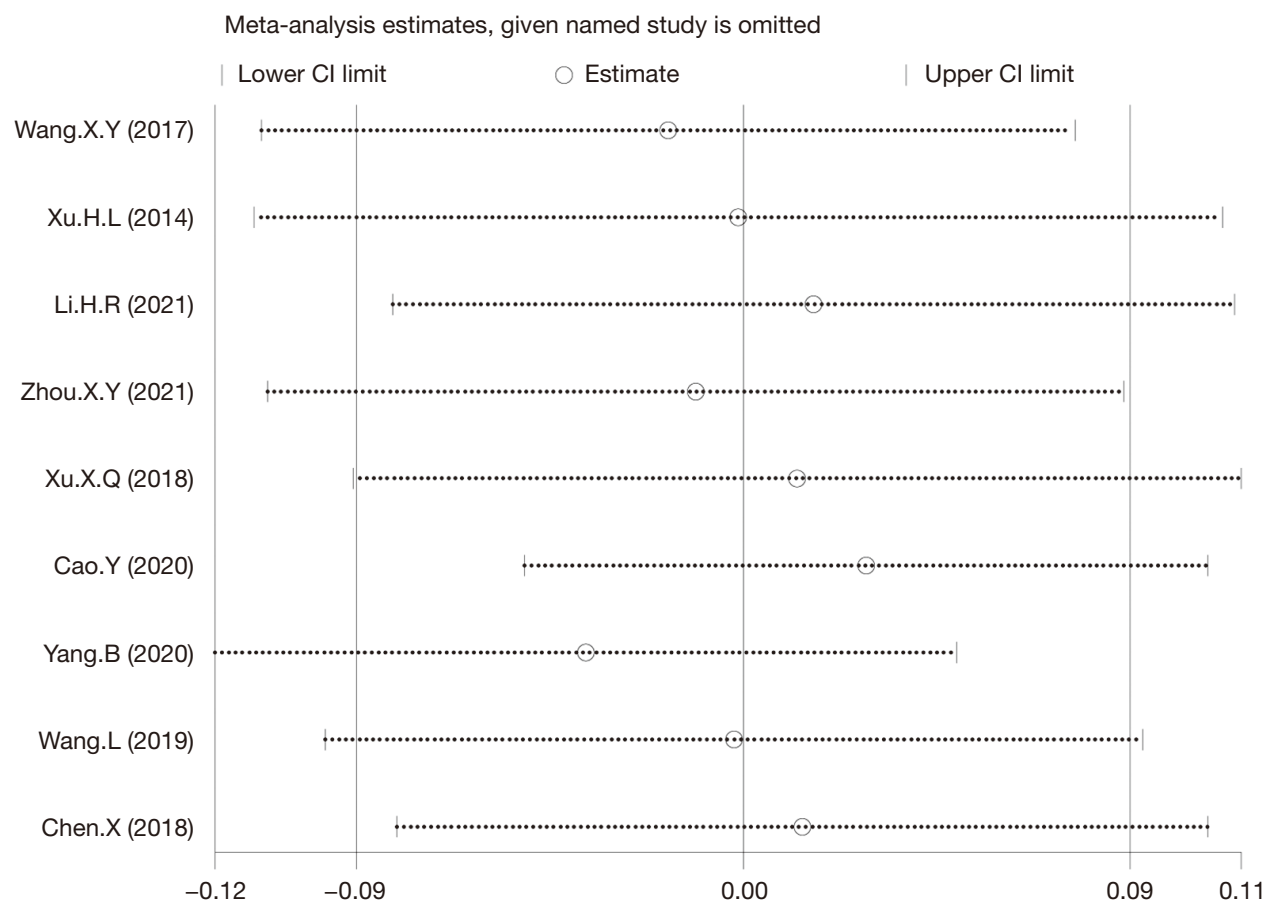

Figure 6 Sensitivity analysis of the included literatures.

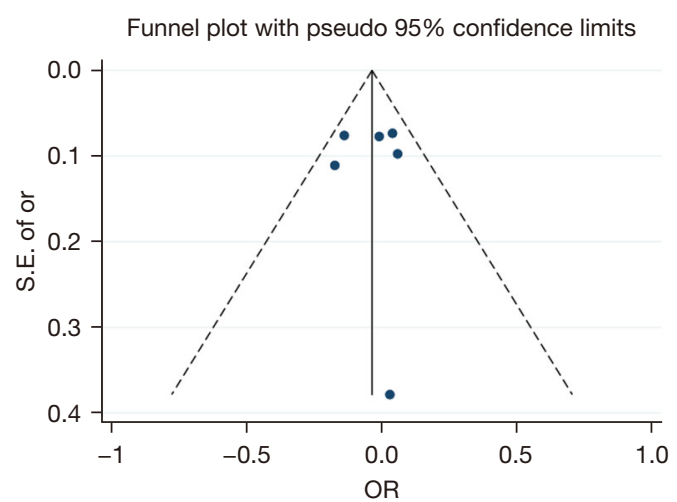

Figure 7 Example 1 of funnel plot.

thyroid nodules in women may be related to menstruation, pregnancy, and other factors related to endocrine disorders, resulting in increased demand for thyroid hormones (32). In addition, estrogen levels may decrease as a woman ages, and serum uric acid levels are negatively correlated with estradiol levels. Indeed, following estrogen replacement therapy, blood uric acid levels may show a significant decrease $(33,34)$. Therefore, the incidence of thyroid nodules may differ significantly between males and females, and the specific mechanisms warrant further analysis.

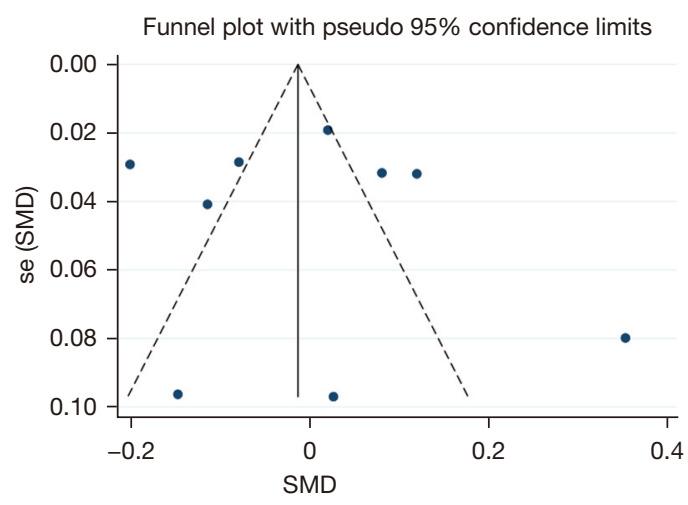

Figure 8 Example 2 of funnel plot.

There were several limitations in this study. First, there is a paucity of studies examining the correlation between hyperuricemia and thyroid nodules, with most of the included literatures being from China. Therefore, the total number of included studies in this meta-analysis was small. Second, there were large variations in the sample sizes and a large gap in the quality of the literature. Due to the language limitation during the literature selection process, there may be certain publication bias. It should be noted that since meta-analysis involves the re-analysis 
of numerous studies, the effectiveness and authenticity of the results are closely related to the quality of the original studies.

\section{Conclusions}

In summary, there was no correlation between hyperuricemia and the incidence of thyroid nodules in the overall population. Interestingly, while hyperuricemia was identified as a protective factor for thyroid nodules in men, it was found to be a risk factor in females. However, since the sample size of this study was limited, further research is warranted to verify the correlation between hyperuricemia and thyroid nodules.

\section{Acknowledgments}

Funding: None.

\section{Footnote}

Reporting Checklist: The authors have completed the PRISMA reporting checklist (available at https://dx.doi. org/10.21037/gs-21-722).

Conflicts of Interest: All authors have completed the ICMJE uniform disclosure form (available at https://dx.doi. org/10.21037/gs-21-722). The authors have no conflicts of interest to declare.

Ethical Statement: The authors are accountable for all aspects of the work in ensuring that questions related to the accuracy or integrity of any part of the work are appropriately investigated and resolved.

Open Access Statement: This is an Open Access article distributed in accordance with the Creative Commons Attribution-NonCommercial-NoDerivs 4.0 International License (CC BY-NC-ND 4.0), which permits the noncommercial replication and distribution of the article with the strict proviso that no changes or edits are made and the original work is properly cited (including links to both the formal publication through the relevant DOI and the license). See: https://creativecommons.org/licenses/by-nc-nd/4.0/.

\section{References}

1. Chen YW, Zhang JB, Ji JY, et al. Clustering and regression analysis of thyroid nodules detection rate in the health examination population in Beijing. International Journal of Epidemiological Infectious Diseases 2014;41:313-6.

2. $\mathrm{Xu} \mathrm{HB}, \mathrm{Xu} \mathrm{CY}$, Zhang $\mathrm{H}$. Investigation and analysis of patients with thyroid nodules present in Chengdu health examination population. Southwest National Defense Medicine 2015;25:1041-2.

3. Han Q, Huang HL. Advances in influencing factors affecting thyroid disease. China Vocational Medicine 2015;42:345-7.

4. Xu HL, Wei J, LM, et al. 11 Survey of the prevalence of thyroid nodules in 11268 health examination population. Disease surveillance 2014;26:987-91.

5. Liu $Y$, Lin Z, Sheng C, et al. The prevalence of thyroid nodules in northwest China and its correlation with metabolic parameters and uric acid. Oncotarget 2017;8:41555-62.

6. Zhou X, Li Y, Jiang Y, et al. Study on the relationship between thyroid nodules and metabolic indicators in the health examination population. Sichuan Medicine 2021;5:483-7.

7. Li HR, Yao Y, Zhang Q. Analysis of the prevalence of thyroid nodules and related factors among 2995 healthy adults in Xiangyang City. Henan Journal of Preventive Medicine 2021;3:194-6.

8. Cao Y. Study of early risk factors for thyroid nodules. Chongqing Medical University 2020.

9. Yang B, Tian YJ, Han FZ. Analysis of thyroid nodules detection and related factors. Southeast National Defense Medicine 2020;2:222-4.

10. Liang YJ, Yu SY, Li SH, et al. Correlation studies of thyroid nodules and the metabolic syndrome. International Journal of Endocrine and Metabolism 2015;35:293-7.

11. Wang L. Analysis of thyroid nodules and nursing effect in health examination. Medical Care 2019;4:165-7.

12. Xu XQ, Jiang YB, Liu M, et al. Analysis of thyroid nodules disease and related factors in the health examination population. Chinese Journal of Health Medicine 2018;3:210-2.

13. Chen X, Yao QH. Association of metabolic syndrome and thyroid nodules and factors influencing thyroid nodules. Journal of Clinical Rational Medications 2018;18:130-2.

14. Ding X, Xu Y, Wang Y, et al. Gender Disparity in the Relationship between Prevalence of Thyroid Nodules and Metabolic Syndrome Components: The SHDCCDPC Community-Based Study. Mediators Inflamm 2017;2017:8481049.

15. Dong LY, He XD, Liu XY, et al. Study on the prevalence 
of Uyghur thyroid nodules in Xinjiang. General Practice in China 2017;11:1314-9.

16. Wang XY, Wang YJ, Artemisia Flower, et al. Clinical study of thyroid nodules and metabolic syndrome. Journal of Xi'an Jiaotong University (Medical Edition) 2017;1:151-3.

17. Jiang H. Investigation on the epidemiological characteristics of thyroid nodules and related factors in Beijing community population. The PLA School of Medicine 2014.

18. Liu SY. Retrospective investigation of 1098 thyroid nodules in Anhui power grid workers. China Nursing Medicine 2014;3:252-3.

19. Xu HL, Cui Y, Lan M, et al. Analysis of factors affecting thyroid nodules in health examination population and health education intervention. Journal of Nurses 2013;18:1662-4.

20. Chen XY. Subsequent factors influencing the prevalence of thyroid nodules and their correlation with various components of the metabolic syndrome. Dalian Medical University 2013.

21. Liu Y, Fan Y, Liu Q, et al. Sex-specific association of serum uric acid dynamics with the incidence of metabolic syndrome in a health check-up Chinese population: a prospective cohort study. BMJ Open 2020;10:e035289.

22. Huo LS, Liu FY, Gao C, et al. Meta-analysis of thyroid nodules prevalence in mainland China. Journal of Hebei Medical University 2017;38:138-41, 205.

23. Yan N. Preliminary study on the prevalence trend of hyperuricemia in Chinese mainland adults from 19852018. Peking Union Medical College 2018.

24. Johnson RJ, Gaucher EA, Sautin YY, et al. The planetary biology of ascorbate and uric acid and their relationship with the epidemic of obesity and cardiovascular disease. Med Hypotheses 2008;71:22-31.

25. Fabbrini E, Serafini M, Colic Baric I, et al. Effect of plasma uric acid on antioxidant capacity, oxidative stress, and insulin sensitivity in obese subjects. Diabetes 2014;63:976-81.

Cite this article as: $\mathrm{Hu} \mathrm{J}$, Luo Y, Lin X. A systematic review and meta-analysis of the correlation between hyperuricemia and thyroid nodules in adults. Gland Surg 2021;10(12):3324-3333. doi: $10.21037 / g s-21-722$
26. Lassoued S, Mseddi M, Mnif F, et al. A comparative study of the oxidative profile in Gravest disease, Hashimoto, sthy roiditis, and papillary thyroid cancer. Biol Trace Elem Res 2010;138:107-15.

27. Itahana $\mathrm{Y}, \mathrm{Han} \mathrm{R}$, Barbier $\mathrm{S}$, et al. The uric acid transporter SLC2A9 is a direct target gene of the tumor suppressor p53 contributing to antioxidant defense. Oncogene 2015;34:1799-810.

28. Martinon F, Pétrilli V, Mayor A, et al. Gout-associated uric acid crystals activate the NALP3 inflammasome. Nature 2006;440:237-41.

29. Tong NW, Xing XP. Handbook of Endocrinology. Beijing: People's Health Publishing House 2015:170-6.

30. Liu M, He Y, Jiang B, et al. Association between serum uric acid level and metabolic syndrome and its sex difference in a Chinese community elderly population. The National Center for Cardiovascular Disease, the Chinese Journal of Circulation. Compilation of China Heart Congress 2014 papers. National Center for Cardiovascular Disease, Chinese Journal of Circulation: Chinese Medical Association 2014:2.

31. Yoshitomi R, Fukui A, Nakayama M, et al. Sex differences in the association between serum uric acid levels and cardiac hypertrophy in patients with chronic kidney disease. Hypertens Res 2014;37:246-52.

32. Kung AW, Chau MT, Lao TT, et al. The effect of pregnancy on thyroid nodule formation. J Clin Endocrinol Metab 2002;87:1010-4.

33. Cao YT, Li J, Zhao QZ, et al. Correlation studies of serum estradiol and testosterone with hyperuricemia. Chinese Journal of Laboratory Medicine 2013;36:1131-3.

34. Ichikawa A, Sumino H, Ogawa T, et al. Effects of longterm transdermal hormone replacement therapy on the renin-angiotensin- aldosterone system, plasma bradykinin levels and blood pressure in normotensive postmenopausal women. Geriatr Gerontol Int 2008;8:259-64.

(English Language Editor: J. Teoh) 